\title{
Clinicolaboratory Parameters of Pediatric COVID-19 Patients and Its Correlation with Outcome: A Study at a Teaching Hospital of Northern India
}

\author{
Anita Kumari ${ }^{1}$, Preeti L Rai ${ }^{2}$, Ruchika Bhatnagar ${ }^{3}$, Prem L Prasad ${ }^{4}$, Prakhar Gupta ${ }^{5}$
}

\begin{abstract}
Aims and objectives: The worldwide prevalence of pediatric coronavirus disease-2019 (COVID-19) infections has risen from 1-5\% in early part of 2020 to $8-9 \%$ in later half of 2020. Though clinical features of pediatric COVID-19 are well researched, its correlation with biochemical parameters and outcome is less evaluated.

Materials and methods: A retrospective evaluation of records of a cohort of 45 children with RT-PCR confirmed COVID-19. We collected information on clinical, laboratory features, and their outcome in all children (age <18 years) admitted between March 1, 2020, and December 31, 2020.

Results: The mean age of the study population was $11.7 \pm 4.83$ years. The average duration of hospital stay of patients was $7.8 \pm 2.51$ days. The majority of COVID-19 positive children were asymptomatic $(20,44.4 \%), 12(26.6 \%)$ had upper respiratory tract features while only $4(8.8 \%)$ had features of lower respiratory tract infection. Five children had nonspecific manifestations. The abnormal chest X-ray findings, the RFT, LFT, and inflammatory markers like C-reactive protein (CRP) (>1 mg/dL), lactate dehydrogenase (LDH) $(>450 \mathrm{IU} / \mathrm{L})$, serum ferritin $(>300 \mathrm{ng} / \mathrm{mL})$, and D-dimer values of $>1,000 \mathrm{ng} / \mathrm{mL}$, in ICU and non-ICU patients were highly significant.

Conclusion: Coronavirus disease 2019 in Indian children seems to affect the older age group. In India, COVID-19 is more associated with comorbidities like chronic kidney disease and chronic infection like tuberculosis rather than congenital heart disease and other acute respiratory illnesses in western studies. Inflammatory markers correlate well with the severity of illness but TLC, DLC, and NL ratio showed variable results and does not correlate with severity of illness.
\end{abstract}

Keywords: Biochemical parameters, Clinical features, ICU admission, Pediatric coronavirus disease 2019.

Pediatric Infectious Disease (2022): 10.5005/jp-journals-10081-1327

\section{INTRODUCTION}

Coronavirus disease-2019 (COVID-19) is caused by severe acute respiratory syndrome coronavirus 2 (SARS-CoV-2). To date, this infection is a posing threat to mankind despite vaccine development at the earliest. As of September 2020, worldwide pediatric population account for $8.5 \%$ of diagnosed COVID-19 cases. ${ }^{1}$ Till the first week of April 2021, 12,801,785 people of India have acquired the infection. ${ }^{2}$ According to the age distribution of COVID-19 patients in India, the incidence is 3.90 and $7.99 \%$ among 0-10 and 11-20 years, respectively. ${ }^{3}$ In India, despite relatively low rates, it is a growing concern that children and adolescents be protected from COVID-19 as they constitute a large proportion of the population. On literature review of available data, it has been seen that when compared with adult patients, pediatric patients have milder clinical characteristics, disease progression, and outcome. The most common symptoms associated with COVID-19 are fever, cough, dyspnea, expectoration, headache, myalgia, and fatigue. Although these signs and symptoms may occur at any time during the overall disease course, children with COVID-19 may not initially present with fever and cough as often as adult patients. ${ }^{4}$

The most common laboratory values reported in various studies are white blood cell count, platelet count, erythrocyte sedimentation rate, C-reactive protein (CRP), urea, creatinine, alanine aminotransferase, and aspartate aminotransferase. ${ }^{5}$ Four serum inflammatory markers which are frequently studied are D-dimer, procalcitonin, creatine kinase, and interleukin- $6{ }^{6}$ Although heterogeneity varied between the parameters analyzed, but trended higher for inflammatory biomarkers.
${ }^{1-5}$ Department of Pediatrics, Shri Ram Murti Smarak Institute of Medical Sciences, Bareilly, Uttar Pradesh, India

Corresponding Author: Anita Kumari, Department of Pediatrics, Shri Ram Murti Smarak Institute of Medical Sciences, Bareilly, Uttar Pradesh, India, Phone: +91 8979837637, e-mail: anitaneo@gmail.com

How to cite this article: Kumari A, Rai PL, Bhatnagar $\mathrm{R}$, et al. Clinicolaboratory Parameters of Pediatric COVID-19 Patients and Its Correlation with Outcome: A Study at a Teaching Hospital of Northern India. Pediatr Inf Dis 2022;4(1):6-10.

Source of support: Nil

Conflict of interest: None

To date, although data on clinical features of COVID-19 in children may be found in plenty on laboratory parameters and its correlation with clinical features and outcome remain scarce. Most published data originate from China Europe and the US, which cannot necessarily be extrapolated to children in India and elsewhere.

\section{Materials and Methods}

We retrospectively reviewed records of 43 children confirmed with SARS-COV-2, who were managed in Shri Ram Murti Smarak Institute of Medical Sciences from March 1, 2020, to December 31, 2020. Coronavirus disease 2019 pediatric cases were defined as a patient in whom SARS-CoV-2 was detected on nasopharyngeal swab specimens using RT-PCR. All confirmed COVID-19 cases $<18$ 
years were included. A standardized data collection spreadsheet was used to collect and record data regarding epidemiological, demographical, clinical symptoms, laboratory measurements, imaging findings, management, and outcome from the case files and the documents of SARS-CoV-2 infected patients. Pyrexia was defined as a body temperature of at least $38.0^{\circ} \mathrm{C}$. Diagnosis of upper respiratory tract infection was based on clinical signs and symptoms, encompassing any of the following: coryza, pharyngitis, tonsillitis, otitis media, or sinusitis. Lower respiratory tract infection was based on clinical signs and auscultation findings. Patients were categorized into asymptomatic, mild, moderate, and severe types as per standard definitions. ${ }^{7}$

\section{Laboratory Investigations}

Routine Investigations

Complete blood count with differential count, renal function test, liver function test, coagulation profile, urine routine, and microscopy. Advanced investigations as CRP, procalcitonin, creatine phosphokinase (CPK), lactate dehydrogenase (LDH), D-dimer, serum ferritin, and interleukin-6 (IL-6) were recorded wherever done. Various laboratory parameter abnormalities were defined as:

- Anemia-Hemoglobin $<10 \mathrm{~g} \%$

- Leukopenia-Total leukocyte count $<4,500 / \mathrm{mm}^{3}$

- Thrombocytopenia-platelet count $<1.5 \mathrm{lakh} / \mathrm{mm}^{3}$

- Leukocytosis-Total leukocyte count $>12,000 / \mathrm{mm}^{3}$

- Neutrophil to lymphocyte ratio $>17$

- Serum ferritin $\rightarrow 150 \mathrm{ng} / \mathrm{mL}$

- D-dimer $\rightarrow 1,000 \mathrm{ng} / \mathrm{mL}$

- $\quad \mathrm{CRP} \rightarrow 1 \mathrm{mg} / \mathrm{dL}$

- $\mathrm{LDH} \longrightarrow 450 \mathrm{U} / \mathrm{L}$

- Procalcitonin-Normal level is taken as $0.1-0.49 \mathrm{ng} / \mathrm{mL}$

- Interleukin-6-Normal level is taken as 0-7 pg/mL

Radiological investigations like chest X-ray were collected and interpretation recorded. Contrast-enhanced computed tomography (CECT) chest if done was also recorded.

The treatment and the various drugs used in treating patients were recorded and analyzed. The guidelines released by the ministry of health and family welfare for suspecting, diagnosing, managing, and discharging COVID-19 disease patients were strictly followed. The outcome was recorded as the requirement of intensive care, recovery, or death.

The Ethical Committee of the Institute has received in-depth that no ethical issue is involved which prevent from conducting the proposed research work. Simple statistical analysis was used to focus on demographic patterns in children pertaining to continuous and categorical variables as appropriate.

\section{Results}

A total of 74 cases aged $<18$ years, of suspected COVID-19 infection, were reported in SRMSIMS (March 1, 2020, till November 30, 2020). Out of which 45 had PCR-confirmed SARS-CoV-2 infection. The mean age of the study population was $11.7 \pm 4.83$, ranging from 1 day to 18 years (Table 1). It has been observed that age was nonnormally distributed in $<1$ year of age group. The percentage of children $<1$ year was $15.5 \%$ of total COVID cases, but out of 7 cases, 4 were newborns (57.1\%). The sex ratio was 0.9:1. Most of the children had exposure to household members with confirmed COVID-19. The average duration of hospital stay of patients was $7.8 \pm 2.51$ days. Four cases (8.8\%) required admission in ICU. In 38 (84.4\%) cases, there were no preexisting medical conditions. In the remaining 7 (15.5\%) cases, children had comorbidities as chronic kidney disease (CKD)-2, chronic liver disease (CLD)-1, tuberculous meningitis (TBM)1, nephrotic syndrome (NS)-1, abdominal tuberculosis-1, hypoxic encephalopathy (HIE)-1.

Although the majority of patients $<18$ years were asymptomatic $(20,44.4 \%)$, pyrexia was the most common symptom at presentation in symptomatic patients, observed in 18 (40\%) individuals (Fig. 1). Cough (26.6\%) was the second most common symptom at presentation. Twelve (26.6\%) had upper respiratory tract features while only four (8.8\%) had features of lower respiratory tract infection, five children had nonspecific manifestations, out of which three children had symptoms and signs of their comorbidities but no features suggestive of primary COVID-19 infections per se. Only one COVID-19 positive child had hypoxemia measured by pulse oximetry at the time of presentation. Chest X-ray was done in all 25 symptomatic cases $(55.5 \%)$ of patients $<18$ years of age. Of those, four $(16 \%)$ had changes consistent with pneumonia. Contrastenhanced computed tomography (CECT) was done in only two cases and none had features suggestive of COVID-19.

Table 1: Demographic characteristic of children with COVID-19

\begin{tabular}{lc}
\hline Male:female ratio & $22: 23(0.9: 1)$ \\
Age (mean with SD) & $11.7 \pm 4.83$ \\
$<1$ year & $7(15.5 \%)$ \\
$1-6$ years & $4(8.8 \%)$ \\
$7-10$ years & $7(15.5 \%)$ \\
$11-18$ years & $27(60 \%)$ \\
Mean duration of stay & $7.8 \pm 2.51$ \\
Comorbidities (CKD, CLD, cerebral palsy, & $7(15.5 \%)$ \\
nephrotic syndrome, TBM) & \\
Severity of illness & \\
Asymptomatic & $19(42.2 \%)$ \\
Mild & $21(46.6 \%)$ \\
Moderate & $1(2.2 \%)$ \\
Severe & $4(8.8 \%)$ \\
Outcome & \\
Recovered & $44(97.7 \%)$ \\
Death & $1(2.2 \%)$ \\
Lama & 0 \\
\hline
\end{tabular}

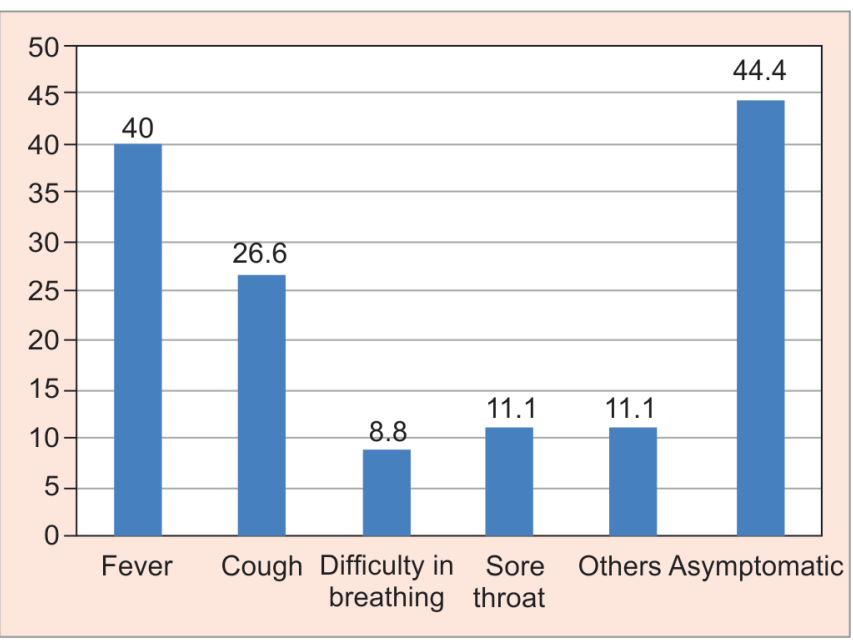

Fig. 1: Frequency of symptoms in children with confirmed COVID-19 
Table 2: Laboratory parameters in children with COVID-19

\begin{tabular}{llcccl}
\hline S.no. & Parameters & Highest & Lowest & Median & Done in cases $(N)$ \\
\hline 1 & Hemoglobin $(\mathrm{g} / \mathrm{dL})$ & 20.2 & 7.2 & 12.14 & 45 \\
2 & Leukocyte count $\left(\mathrm{mm}^{3}\right)$ & 30,800 & 3,500 & 6,500 & 45 \\
3 & Lymphocytes (\%) & 67 & 02 & 30 & 45 \\
4 & Sr. ferritin & 2,500 & 11.7 & 35.5 & 22 \\
5 & D-dimer $(\mathrm{ng} / \mathrm{mL})$ & 13,300 & 312 & 486.5 & 29 \\
6 & CRP $(\mathrm{mg} / \mathrm{dL})$ & 20.6 & 0.6 & 0.6 & 21 \\
7 & LDH & 954 & 219 & 289 & 16 \\
8 & PCT & 3.4 & 1 & 2.2 & 02 \\
9 & IL-6 & 419.96 & 2.4 & 13.89 & 03 \\
\hline
\end{tabular}

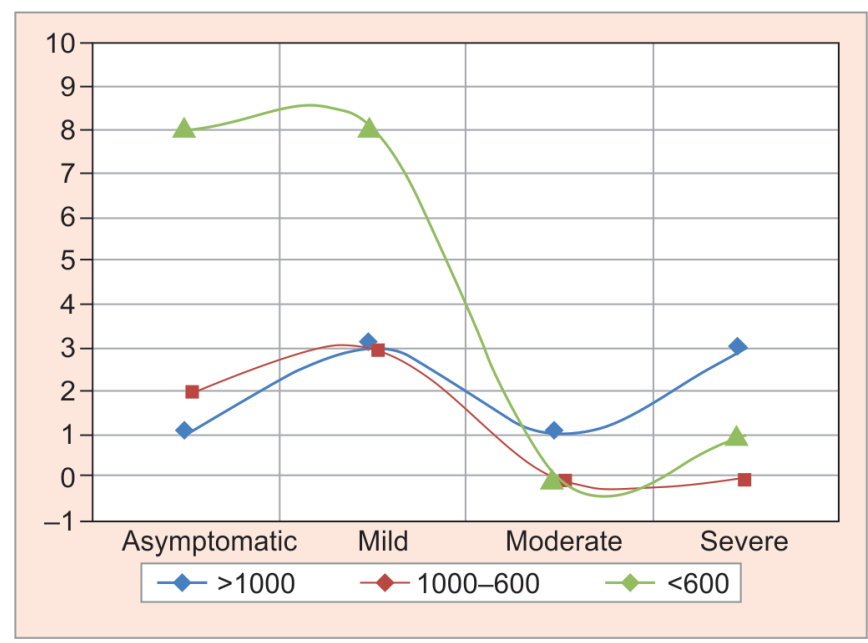

Fig. 2: Correlation of D-dimer levels with severity of illness

Lots of variability was seen in the abnormalities of laboratory parameters as well as in organ system involvement in patients of COVID-19 disease (Table 2). A significant neutrophil-lymphocyte ratio was observed in one of the patients. C-reactive protein was done in 21 cases but it was raised only in 4 (23.8\%) patients. Liver function test and renal function test were done in $53.3 \%$ and $73.3 \%$, respectively. Deranged LFT and RFT were seen in 1 (4.1\%) and 4 $(12.1 \%)$ cases, respectively. C-reactive protein (46.6\%), D-dimer (64.4\%), lactate dehydrogenase (35.5\%), and serum ferritin (48.8\%) were done in all symptomatic cases, and in addition to that, it has been done in few asymptomatic cases also as reflected in Table 3. Abnormality in organ system parameters and inflammatory markers of COVID-19 is shown in Table 3. Correlating the D-dimer levels with disease severity reflects that it is not significant ( $p=$ 0.37929), although Figure 2 very well reflects that $D$-dimer values could remain elevated even in mild and asymptomatic patients. Table 4 depicts various treatment modalities and their frequencies, given to the patients. On comparing the demographic data of ICU admissions and non-ICU admissions, we got an odds ratio and a $p$ value which was insignificant for the basic demographic characteristics, i.e., for age and gender, in both the ICU and non-ICU group. However, the comparison of associated comorbidities with COVID-19, the abnormal chest X-ray findings, the RFT, LFT, and inflammatory markers like CRP ( $>1 \mathrm{mg} / \mathrm{dL}), \mathrm{LDH}(>450 \mathrm{IU} / \mathrm{L})$, serum ferritin (>300 ng/mL), and D-dimer values of $>1,000 \mathrm{ng} / \mathrm{mL}$, in ICU and non-ICU patients were highly significant (Table 5).
Table 3: Abnormality in laboratory parameters pertaining to organ systems and inflammatory markers of COVID-19

\begin{tabular}{clcc}
\hline S. no. & Abnormal parameter & $N$ & $\%$ \\
\hline 1 & Anemia $(<10 \mathrm{~g} \%)$ & 4 & 8.8 \\
2 & Leukopenia $\left(<4,000 \mathrm{~mm}^{3}\right)$ & 2 & 4.4 \\
3 & Lymphopenia $(15 \%)$ & 3 & 6.6 \\
4 & Thrombocytopenia $(<1.5$ lakh) & 4 & 8.8 \\
5 & Leukocytosis $(>12,000)$ & 6 & 13.3 \\
6 & NL ratio $(>17)$ & 1 & 2.2 \\
7 & Sr. ferritin $(>300 \mathrm{ng} / \mathrm{mL})(22)$ & 7 & 31.81 \\
8 & D-dimer $(>1,000 \mathrm{ng} / \mathrm{mL})(29)$ & 8 & 27.58 \\
9 & LDH $(>450)(16)$ & 3 & 18.75 \\
10 & CRP $(>1 \mathrm{mg} / \mathrm{dL})(21)$ & 5 & 23.80 \\
11 & Deranged LFT (24) & 1 & 41.67 \\
12 & Deranged RFT (33) & 4 & 12.12 \\
13 & Chest X-ray (45) & 4 & 16 \\
14 & CECT thorax (2) & 0 & 0 \\
\hline
\end{tabular}

Table 4: Type of treatment given to positive COVID-19 children

\begin{tabular}{ll}
\hline Type of treatment & Numbers (\%) \\
\hline $\begin{array}{l}\text { 1. Supportive treatment (vitamin C, } \\
\text { zinc, } N \text {-acetylcysteine) }\end{array}$ & $34(75.5)$ \\
2. Fabiflu & $2(4.4)$ \\
3. Remdesivir & $1(2.2)$ \\
4. Hydroxychloroquine & $7(15.5)$ \\
5. Azithromycin & $23(51.1)$ \\
6. Ivermectin & $12(26.6)$ \\
7. Doxycycline & $12(26.6)$ \\
8. Toclizumab & 0 \\
9. Oseltimavir & $1(2.2)$ \\
10. Dexamethasone & $4(8.8)$ \\
11. Methylprednisolone & $2(4.4)$ \\
12. Enoxaparin & $7(15.5)$ \\
13. Vassopressors & $1(2.2)$ \\
14. Plasma therapy & 0 \\
Combination therapy & \\
15. (Doxycycline + ivermectin) & $9(20)$ \\
16. (Azithromycin + & $7(15.5)$ \\
hydroxychloroquine) & \\
\hline
\end{tabular}




\begin{tabular}{|c|c|c|c|c|}
\hline Characteristics & ICU admission $(N=4)$ & Non-ICU admission $(N=41)$ & Odds ratio & $p$ value \\
\hline Male & 3 & 19 & 3.4737 & 0.2980 \\
\hline Female & 1 & 22 & & \\
\hline Age & 0 & 21 & 5.0000 & 0.1484 \\
\hline$<1$ year & 2 & 05 & & \\
\hline $1-6$ years & 0 & 04 & & \\
\hline $7-10$ years & 0 & 07 & & \\
\hline $11-18$ years & 2 & 25 & & \\
\hline Comorbidities (7) & & & 0.0585 & 0.0016 \\
\hline Chronic kidney disease (2) & 1 & 1 & & \\
\hline Chronic liver disease (1) & 1 & 0 & & \\
\hline Cerebral palsy (1) & 1 & 0 & & \\
\hline Nephrotic syndrome (1) & 0 & 1 & & \\
\hline Tuberculous meningitis (1) & 1 & 0 & & \\
\hline Abdominal tuberculosis (1) & 0 & 1 & & \\
\hline Abnormal chest X-ray findings (4) & 2 & 2 & 0.0976 & 0.0392 \\
\hline Raised D-dimer value (>1,000 ng/mL) & 3 & 5 & 0.1626 & 0.0433 \\
\hline Sr. ferritin (>300 ng/mL) & 4 & 3 & 13.6667 & 0.0048 \\
\hline $\mathrm{LDH}(>450 \mathrm{IU} / \mathrm{L})$ & 3 & 0 & 64.5556 & 0.0088 \\
\hline $\mathrm{CRP}(>1 \mathrm{mg} / \mathrm{dL})$ & 4 & 1 & 41.0000 & 0.0026 \\
\hline Deranged LFT & 1 & 0 & 27.6667 & 0.0517 \\
\hline Deranged RFT & 4 & 0 & 83.0000 & 0.0049 \\
\hline
\end{tabular}

\section{Discussion}

Children and adolescents who were admitted and treated within the hospital settings served as the primary source of data in the present study. The mean age of children in the present cohort was $11.7 \pm 4.83$ years which is higher than various Chinese and European studies but comparable to a study from the USA who had reported higher median age (11 years). ${ }^{8-10}$ Similarly, $60 \%$ of all pediatric COVID-19 cases of the present study were adolescent as that of a study from the USA. ${ }^{10}$ However, the age distribution was not uniform in an infant $<1$ year of the present study, similar to a study from Europe but unlike an American study. ${ }^{9}$ There was no sex predilection in the present study like various other studies. ${ }^{5,11}$ The average duration of hospital stay of patients in our study was $7.8 \pm$ 2.51 days. Similar to ours, in a study from Canada on characteristics and outcomes of children with COVID-19, the total duration of hospital stay and PICU ranged from 4 to 13 days and 3 to 9 days, respectively. ${ }^{12}$ In the present study, comorbidities were present in $15.5 \%$ of cases. Unlike ours, a study on COVID-19 in children from Lancet had observed comorbidities in $25 \%$ of children. ${ }^{9}$ Similarly, in the meta-analysis of pediatric COVID-19 related studies from developed countries, underlying medical conditions and coinfection were noted in a higher number of cases than in this study. ${ }^{6}$ They also noted that most of the comorbidities were of the cardiovascular and respiratory system unlike ours where none had cardiac or respiratory comorbidities; rather chronic kidney disease and tuberculosis were important underlying conditions. ICU admission rate was $8.8 \%$ in the present study, concordance with various national and international studies where it ranges from 0.4 to $8 \% .{ }^{10,11}$ Fever and cough, like the majority of studies, were the commonest clinical features in symptomatic patients through percentage varies. In concordance with other Indian studies, near half of the total patients (42.2\%) were asymptomatic in our study. ${ }^{11,13}$ However, in studies from developed countries it is lower (16-21\%). ${ }^{9,14}$ Only a quarter of children, of present study had features of URTI while LRTI was noted only in $8.8 \%$ cases but unlike this the incidences of both were higher (URTI-50\%, LRTI-25\%) in European pediatric COVID-19 study. ${ }^{9}$ This could be due to variation in host response, immunity status, race, ethnicity, and various environmental/geographical factors which play key factors in manifestations of the disease.

From various studies, it has been noted that there is less derangement of the hematologic and biochemical parameters in children than adults. Lymphopenia in adults has been correlated with increased risk of mortality; however, neither lymphopenia nor high mortality was seen in COVID positive children of ours neither in other pediatric COVID-19 related studies. ${ }^{6,15}$ It may be due to the relative immaturity of the immune system in young children, as well as differences in viral susceptibility/response to infection, which possibly explains the differences in laboratory trends seen in the pediatric vs the adult population of COVID-19 patients. Similar to a pooled analysis and review of laboratory parameters in pediatric COVID-19 studies, ours laboratory profile, also showed an inconsistent pattern of total leukocyte, differential leukocyte count, and N/L ratio in mild, moderate, and severe cases. ${ }^{6}$ This inconsistency raised the question of the utility and reliability of leukocyte index in monitoring disease severity in the pediatric population. Of interest, D-dimer was found to be as frequently elevated in asymptomatic and mild COVID-19 cases as in severe cases but higher levels, i.e., $>1000 \mathrm{~g} / \mathrm{mL}$ were more seen in severe cases while lower high values were observed in asymptomatic and mild cases. However, correlating the D-dimer level with disease severity does not reflect any significance. Similar to ours, the study 
of pooled analysis and review of laboratory parameters in pediatric COVID-19 also observed the increase in D-dimer in both severe and mild cases. ${ }^{6}$ However, they have not mentioned anything regarding levels of $D$-dimer in either type. From the same study, it has been also observed that heterogeneity varied between the parameters analyzed for each patient and it trended higher for inflammatory biomarkers. The same thing has also been observed in the present study, so inflammatory markers like LDH, serum ferritin, and CRP were not assessed for disease severity. However, the same metaanalysis study has observed that children with severe COVID-19 had somewhat consistent trends of elevated LDH, CRP, D-dimer, PT, and PCT levels, similar to adult patients with COVID-19 but these variables similar to the present study have not been consistently measured across studies.

In our cohort, neither age nor gender was associated with an increased likelihood of requiring ICU admission; however, the presence of other comorbid conditions and raised inflammatory markers like CRP, LDH, serum ferritin high value of D-dimer, and abnormal chest X-ray findings were strongly associated with ICU admission. Unlike this in a multinational multicentric study of COVID-19 in children and adolescents from Europe had shown male gender and age $<1$ month, preexisting medical condition, LRTI, radiological findings suggestive of ARDS, and viral coinfection were significantly associated with pediatric COVID-19. ${ }^{9}$ They have not mentioned laboratory parameters and their association with severity or outcome. As in meta-analysis of adult COVID-19 clinicolaboratory parameter assessment study, positive association of impaired liver and renal function tests with the severity of COVID19 was also seen in this study. ${ }^{15}$ The present data also reflects the variations regarding drug treatment options for COVID-19 among treating physicians.

\section{Conclusion}

Coronavirus disease-2019 in Indian children seems to affect the older age group. In India, COVID-19 is more associated with comorbidities like chronic kidney disease and chronic infection like tuberculosis rather than congenital heart disease and other acute respiratory illnesses in western studies. Inflammatory markers correlate well with the severity of illness but TLC, DLC, and NL ratio showed variable results and does not correlate with severity of illness. Further studies are necessary to verify these findings.

\section{ACKNOWLedgment}

We are thankful to the management of SRMSIMS for providing data on pediatric COVID-19 children.

\section{References}

1. Coronavirus disease (COVID19): Schools. World Health Organization. 2020 Sep 18.

2. WHO COVID19 Dashboard 2021. 2021Apr 07.

3. Graphical illustration of data from COVID19 cases in India, National Center for Disease Control (NCDC) 2020. Ministry of Health and Family, Government of India.

4. VDV Coronavirus disease 2019. Caring for children. https://www.cdc. gov/coronavirus/2019-ncov/hcp/pediatric-cp.html. Accessed on 28th May, 2020.

5. Fakiri ELK, Nassih H, Sab Ait l, et al. Epidemiology and clinical features of coronavirus disease 2019 in moroccan children. Indian J Pediatr 2020;57(9):808-810. DOI: 10.1007/s13312-020-1958-8.

6. Henry MB, Benoitb WS, Santos de Oliveir HM, et al. Maria helena santos de oliveira. Laboratory abnormalities in children with mild and severe coronavirus disease 2019 (COVID-19): a pooled analysis and review. Clin Biochem 2020;81:1-8. DOI: 10.1016/j.clinbiochem.2020.05.012.

7. Clinical management protocol: COVID-19. Government of India Ministry of Health and Family Welfare Directorate General of Health Services (EMR Division) Version 5, 03.07.20.

8. Dong $\mathrm{Y}, \mathrm{Mo} \mathrm{X}, \mathrm{Hu} \mathrm{Y}$, et al., Epidemiological characteristics of 2143 pediatric patients with 2019 coronavirus disease in China. Pediatrics. 2020 Mar. Available from https://pediatrics.aappublications.org/ content/pediatrics/early/2020/03/16/peds.2020-0702.1.full-text.pdf. Accessed on April 04, 2020.

9. Götzinger F, Santiago-García B, Noguera-Julián A, et al. COVID-19 in children and adolescents in Europe: a multinational, multicentre cohort study. Lancet Child Adolesc Health 2020;4(9):653-661. DOI: 10.1016/S2352-4642(20)30177-2.

10. Corona virus Disease in Children - United States February 12-April 02 2020. https://www.cdc.gov/mmwr/volumes/69/wr/mm6914e4.htm. Accessed on 28th May, 2020.

11. Sarangi B, Reddy VS, Oswal JS, et al. Epidemiological and clinical characteristics of COVID-19 in Indian children in the initial phase of the pandemic. Indian J Pediatr 2020;57(10):914-917. DOI: 10.1007/ s13312-020-1994-4.

12. Shekerdemian LS, Mahmood NR, Wolfe KK, et al. Characteristics and outcomes of children with coronavirus disease 2019 (COVID19) infection admitted to US and Canadian pediatric intensive care units. JAMA Pediatr 2020;174(9):868-873. DOI: 10.1001/ jamapediatrics.2020.1948.

13. Meena J, Yadav J, Saini L, et al. Clinical features and outcome of SARS CoV-2 infection in children: a systematic review and meta-analysis. Indian Pediatr 2020;57(9):820-826. DOI: 10.1007/s13312-020-1961-0.

14. Christophers B, Marin BG, Oliva R, et al. Trends in clinical presentation of children with COVID-19: a systematic review of individual participant data. Pediatr Res 2020. Online ahead of print 10.1038/ s41390-020-01161-3.

15. Moutchial J, Pokharel P, Kerri A, et al. Clinical laboratory parameters associated with severe or critical novel coronavirus disease 2019 (COVID-19): a systematic review and meta-analysis. PLoS One 2020;15(10):e0239802. DOI: 10.1371/journal.pone.0239802. 